\title{
Direct-Digital Synthesis Using Delta-Sigma Modulated Signals
}

\author{
Yuichiro Orino*, Minoru Kuribayashi Kurosawa* and Takashi Katagiri ${ }^{\dagger}$ \\ * Dept. of Advanced Applied Electronics, Tokyo Institute of Technology, Yokohama, Japan \\ Email: orino@ae.titech.ac.jp, mkur@ae.titech.ac.jp \\ ${ }^{\dagger}$ Sutekina Inc., Komagane city, Nagano, Japan, Email: takashi.katagiri@primemotion.com
}

\begin{abstract}
A new technique that use of DSM signal in a common technique for dgitally synthesizing an arbitrary waveform, which is generally known as direct-digital synthesis (DDS), is described in this paper. In this way, a DDS frequency resolution is not restricted to the phase resolution in DDS and requires no phase truncation technique. As a simulation result, low-pass filtered output waveform is smooth at a lower frequency with respect to clock frequency. At lower frequency, this technique leads to higher spurious-free-dynamic-range than that of ideal linear interpolator output with same phase resolution. Therefore, proposed combination of DSM signal and basic DDS is able to realize high frequency resolution and high spectral-purity at lower frequency band by small circuits.
\end{abstract}

\section{INTRODUCTION}

In recent years, the Delta-Sigma $(\Delta \Sigma)$ modulation is getting popular in analog-to-digital converters (ADCs) and digitalto-analog converters (DACs), because of its advantages in linearity, precision and cost.

Digital signal generated by oversampled Delta-Sigma modulator has both merits of high dynamic range and wide bandwidth as long as it is generated at enough high rate, even though its resolution is low, for instance only 1 bit. Delta-Sigma-modulated (DSM) signal has few noise in low frequency band in the effect of modulation, and it allows have high dynamic range over the limitation of quantization level.

The DSM format is attractive today as a digital signal representation instead of a conventional pulse-code-modulated (PCM) format. There are some commercial applications. For instance, Super Audio CD, new-generation music-delivery format, utilizes a this type of signal format, which labelled as Direct Stream Digital and consists of 1-bit DSM signals.

Now a day, the 1-bit DSM format also attracts attention for use in the digital signal processing. This is a new methodology of processing DSM pluses directly at oversampled rate without converting it into PCM format at Nyquist rate, and which will help decrease hardware complexity and allow wide-band and low-latency processing circuit at low cost.

This methodology has been studied for several purposes, such as filters [1], hardware implementations of pulsed neural networks [3] and digital control systems. Some pure algorithms and circuit designs for fundamental arithmetics have been also proposed in [2] independently of the abovementioned studies.
In the methodology of DSM signal processing, a new application of DSM signal is described in this paper. Specifically, a use of DSM signal in a common technique for dgitally synthesizing an arbitrary waveform, which is generally known as direct-digital synthesis (DDS), is examined.

The remainder of the paper is divided into ....

\section{Conventional Direct-Digital Synthesis}

Direct-digital synthesis (DDS) is a technique for dgitally synthesizing an arbitrary repetitive waveform at the angular rate which is determined by the input signal of DDS. A basic DDS circuit consists of a phase accumulator and a phase-toamplitude converter, which is usually realized by a look-up table (LUT) in which an arbitrary waveform sampled. When sampled waveform is sine form, a DDS circuit can synthesize a sinusoidal wave of an arbitrary frequency.

The basic DDS circuit has trade-off problems inherently between costs and performances. First, there is a cost-quality trade-off. It is desirable to increase a number of amplitude samples and their resolution in a LUT for reasons of output quality, but large circuits are required and greatly increase costs. Second, there is a trade-off between costs and output frequency resolution.

The discussion will focus on the problem between phase resolution and output frequency resolution. The output frequency $f_{\text {out }}$ is determined by the input rate $\Delta P$. The frequency is given by

$$
f_{\text {out }}=f_{c l k} \cdot \frac{\Delta P}{2^{N_{P}}} \quad\left(\Delta P=\left[0,1, \cdots, 2^{N_{P}}-1\right]\right)
$$

where $f_{c l k}$ is a clock frequency and $N_{P}$-bit is a resolution of the phase, which means a bit width of the accumulator. When $\Delta P$ is an constant integer, a DDS circuit generates a constant frequency.

The minimum step of output frequency $\Delta f_{\text {out }}$ can be written as bellow by letting $\Delta P=1$ in (1).

$$
\Delta f_{\text {out }}=\frac{f_{c l k}}{2^{N_{P}}} .
$$

Because the angular rate is specified as a descrete value, the output frequency becomes discrete. Therefore, the frequency resolution is dominated by minimum resolution of the angular rate $\Delta P$, which is equal to the resolution of phase. 


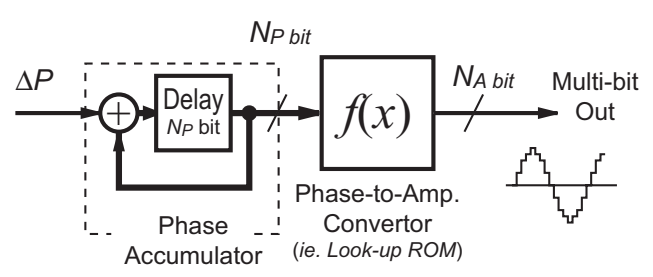

Fig. 1. Diagram of basic DDS circuit.

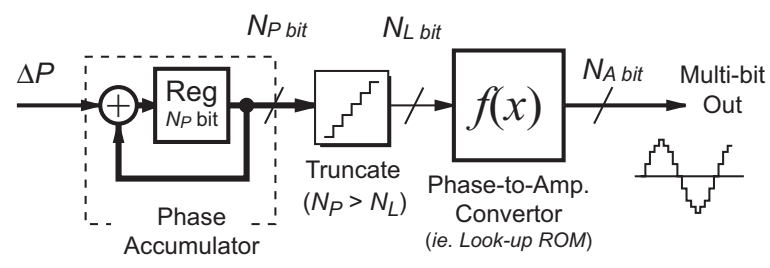

Fig. 2. Diagram of DDS with phase truncation.

To make frequency resolution high, phase resolution requires to be increased. It means that the size of look-up table becomes quite large. In order to reduce a number of samples in LUT keeping a frequency resolution, the technique of phase truncation is usually introduced. Fig. 2 shows a structural diagram of this type of DDS. With this technique, a bit width of the accumulator and a size of table are allowed to configure independently.

However, a phase truncation gives errors in phase and the phase errors result in amplitude errors. When DDS has a sinusoidal waveform, errors causes spurious in spectrum. To ease this problem, some dissolution methods have been proposed, which include the phase dithering technique [4] and the truncation error feedback technique [5]. The phase dithering method is used to randomize truncation errors, and destroys the coherence of spurious components. The nonsubtractive dither, however, increases the resulting noise level. In contrast, the truncation error feedback technique provides effects of randomizing truncation errors and suppressing lowfrequency noise. It is noted that the error feedback technique is equivalent in functionality to the Delta-Sigma modulation. Therefore, it requires that output frequency is enough low with respect to the clock frequency for reason of differential characteristic of modulated noise.

\section{SYNTHESIS USING A DSM SIGNAL}

We propose to utilize a DSM signal for DDS technique in this paper. A DSM signal is used as a rate signal. In conventional way, a rate should be a constant value for a constant frequency, so a rate is simply represented in PCM format. We propose to break this common sense and to use the DSM format in sptead for PCM.

In proposed way, the rate $\Delta P$ is always a vibrating signal even when a constant frequency is generated. The vibration, which is caused by Delta-Sigma modulation, makes it possible to carry a high-resolution information over the limit of apparent resolution. It will result that resolution of $\Delta P$ is equivalently enhanced over LSB limit because that DSM signal can transmit an information with higher quality than its resolution. Thus, it is expected that delicate angular rate and phase itself which can not be represented exactly in finite resolution are able to be realized by DSM signal from a longterm perspective. And it will become possible to enhance frequency resolution without increasing the resolution of the phase accumulator and phase truncation.

The proposed way can be configured with a basic DDS circuit and an auxiliary $\Delta \Sigma$ modulator prior to DDS. But the $\triangle$ SIGMA modulator is not necessarily required when an output of superior circuit is already DSM format, and it can be omitted.

\section{EXAMINATION WITH 1-BIT DSM SIGNAL}

Actual effects of using DSM signal for DDS are tested by computer simulations. Using the input signal generated by additional digital $\Delta \Sigma$ modulator, the output of DDS is examined.

We choose the second-order digital $\Delta \Sigma$ modulator which outputs single-bit stream. The system of this modulator can be expressed generally as

$$
\begin{aligned}
y_{i} & =\text { Quant }\left\{x_{i}-\left(2 e_{i}-e_{i-1}\right)+d_{i}\right\} \\
e_{i+1} & =y_{i}-\left\{x_{i}-\left(2 e_{i}-e_{i-1}\right)\right\}
\end{aligned}
$$

when $y_{i}$ is an output, $x_{i}$ is an input, $e_{i}$ is a quantization error and $d_{i}$ is a non-subtractive dither which is introduced for avoiding a problem of periodic quantization error. Function Quant $(x)$ means quantization.

At the following, 1-bit DSM signals is discussed. The random numbers uniformly distributed within $[-1 / 2,+1 / 2]$ is added prior to the quantizer. A digital Delta-Sigma modulator performs calculations ideally with real numbers, which are digitally represented by 64-bit floating-point notation (IEEE standard 754).

DDS is simply modeled by integer arithmetic without any rounding process and the integer array which roles LUT. In order to only consider problems of phase resolution, the bit width of amplitude samples in LUT is set to 24 bits. It is large enough to deal without considering quantization errors.

Samples in LUT is calculated by the following equation:

$$
A_{n}=V \sin \left(2 \pi \frac{n}{2^{N_{P}}}\right) \quad\left(n=\left\{0,1, \cdots, N_{P}-1\right\}\right)
$$

An amplitude $V$ of sine wave is set to $2^{24-1}-1$ actually, but simulation results are normalized by the amplitude $V$ in below analyses.

\section{A. Time-domain characteristics}

The simulation result of synthesizing with the proposed method is shown in Fig. 3 and 4, when simulation parameters are: the phase accumulator bit-width, which is equal to the LUT phase resolution, $N_{P}=4$ bits and the input phase incremental value $d P=2^{N_{P}} / 10^{5}=1.6 \times 10^{-4}$ constantly, and all delays in the model are set to zero initially. This 


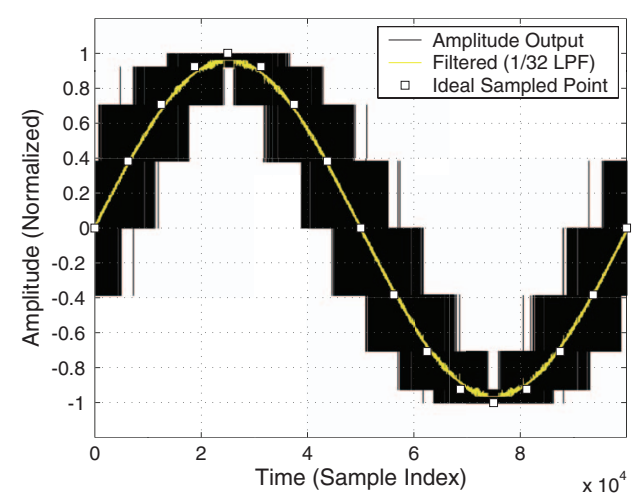

Fig. 3. Output waveform of DDS with 1-bit DSM signal (phase resolution: $\left.N_{P}=4 \mathrm{bit}\right)$.

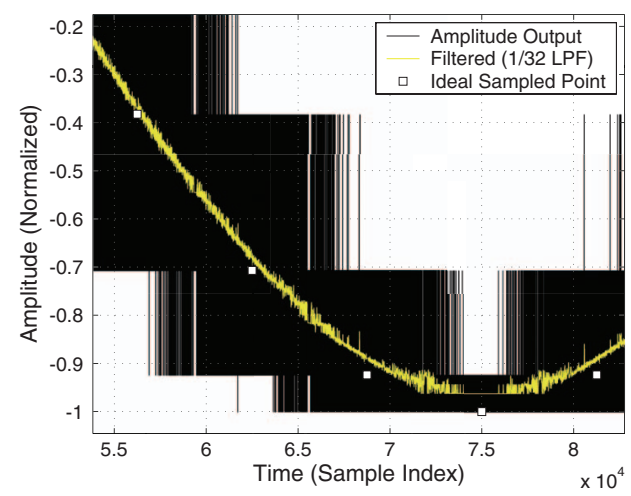

Fig. 4. Zoom of Fig.3

extremely low phase resolution of 4 bits was chosen to emphasize the expected effort of the DSM signal. Moreover, it is noted that the value of $d P$ is less than least significant bit (LSB) of phase, allowing a synthesis at low frequencies beyond the restriction of finite bit-width.

In fig. 3 and 4, not only the raw output signal, but also the low pass filtered plot of the output is shown. The low pass filter cutoff frequency is $f_{c l k} / 64$. This is because that components of $\Delta \Sigma$-modulation noise in higher frequencies are diminished to pay attention to only meaningful components mainly exist in lower frequency band. Consequently, it is important that the cutoff frequency is enough high not to cut off the meaningful components. The cutoff frequency we use is sufficiently valid. Fig. 3 shows that the filtered signal is a nearly sine waveform, not form of stairs. Although details of the filtered output shows that there are certain deformations at each crest of the sine wave and the normalized amplitude of wave is slightly less than 1, the low pass filtering to DDS output in proposed way can yield a fine smoothed sine wave, as in the case of dithering in truncation.

\section{B. Spectral Characteristics}

Fig. 5 shows the frequency spectrum of the DDS output in the band of interest, which is estimated by discrete Fourier

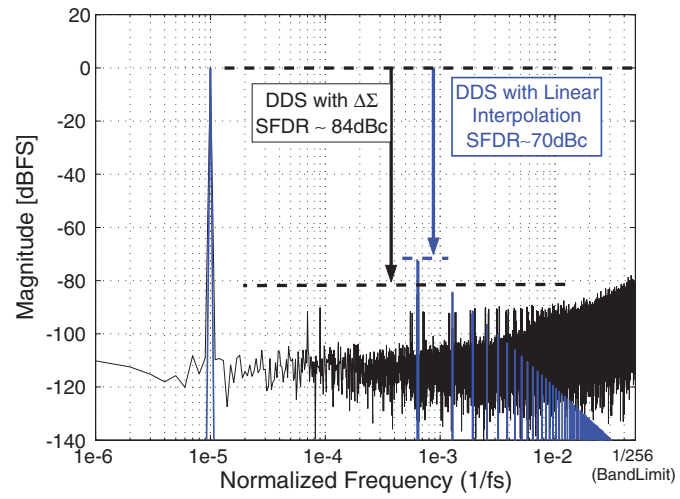

Fig. 5. Power spectra of proposed DDS output and linear-interpolated output. (phase resolution: $N_{P}=6$ bit)

transform (DFT). In this simulation, the phase resolution of $N_{P}=6$ bit was selected.

From Fig. 5, it is shown that some harmonic components which are related with the output frequency and the phase resolution are present, but these are quite small considering its low phase resolution. Main components of spurs are harmonics of wanted frequency, especially 63rd and 65th harmonic. Considering that 63 is equal to $2^{N_{P}}-1$ and 65 is equal to $2^{N_{P}}+1$, this is the effect of aliasing. Although aliasing distortions usually become much larger with low phase resolution, a high spurious-free dynamic range (SFDR) of $84 \mathrm{dBc}$ with a bandwidth of dc to $f s / 256$ is achieved in this case.

For instance, in Fig. 5, the spectral comparison with the result of ideal linear interpolation which uses same number of samples at same clock frequency shows that the proposed method offers the higher SFDR in the band of interest. The SFDR of proposed method is, of course, lower than these of higher order interpolation methods, such like a cubic interpolation, but the proposed method can yield high SFDR without auxiliary interpolation circuit.

These results show that the proposed method allows desirable characteristics of synthesis, such that high frequencyresolution, high spectral-purity and high SFDR at lower frequencies, even with coarse phase resolution.

\section{Order of Modulation}

To test for effect of $\Delta \Sigma$ modulation, we have attempted to use a simplest first-order modulation, not only a second-order. Fig. 6 shows spectra of DDS output in both cases, when inputs are DSM signals of constant $d P=2^{N_{P}} / 10^{5}=6.4 \times 10^{-4}$.

As can be seen in Fig. 6, errors were distributed flatly among whole range of frequencies with first-order modulation. However, with second-order modulation, noises were reduced at low frequencies but increased at high frequencies. Taking notice of the noise level curve in the result with 2nd-order modulation, we can see that the noise level raises by $20 \mathrm{~dB}$ per decade in high frequency band like a spectrum of firstorder DSM signal itself.

So this spectral characteristic of noise is expected to be a 


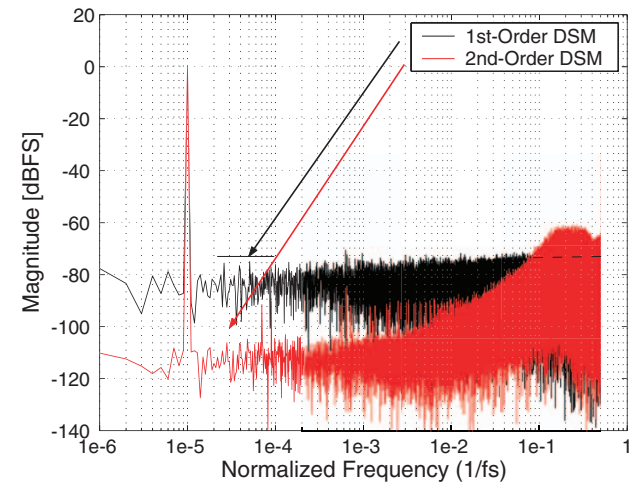

Fig. 6. Comparison of spectra between orders of modulation.

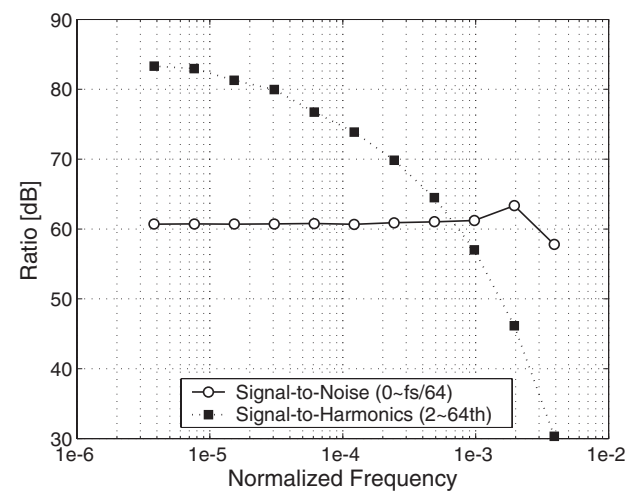

Fig. 7. Frequency characteristics of DDS with 1-bit DSM signal

result of the integral operation by the accumulator. Although the randomly distributed noise in the phase signal will be distorted by the nonlinear function realized as the lookup table, the above-mentioned view may explain the spectral characteristic roughly. As the result, we can say that higher order modulation with oversampling technique is desired for getting high signal-to-noise ratio in the band of interest.

\section{Output Frequency Characteristics}

Figure 7 shows differences in characteristics of signal quality with respect to output frequency. From fig. 7, it is shown that the lower the output frequency is given, the smaller the harmonic distortion becomes as a trend. In contrast, values of SNR (signal-to-noise ratio) are almost same in wide frequency range in spite of higher frequencies than $1 \mathrm{e}-2$. These results are reasonable because harmonics generally have coherence with oscillating frequency and noise power is mainly dominated by Delta-Sigma modulation noise which have no relationship with input signals of the modulation. Although the proposed method has a capability to generate high quality sinusoidal waveforms at lower frequencies with respect to the clock frequency, quality becomes degraded at high frequencies, especially at higher than 1e-2. Therefore, it will be suitable for use in applications which requires a continuously variable frequency in a low frequency domain.
While the low phase resolution of 6 bit is used in the analyses of this paper, using a higher phase resolution will easily increase a signal quality, i.e. SNR and/or SFDR. But in this case, the variable frequency range will also be affected, and be narrowed in some situations. Because of those complicated relations among the phase resolution, the variable frequency range and the signal quality, a performance of this method should be discussed quantitatively in a certain configuration which complies with a practical requirement. This is future work.

\section{CONCLUSIONS}

We have proposed and discussed a synthesizing a sinusoidal wave digitally which uses a $\Delta \Sigma$-modulated signal as a phase rate signal of the basic DDS circuit. The proposed way makes it possible to realize delicate angular rates under the minimum limit of the digital representation, so there are no reasons for increase a resolution of the phase accumulator in order to enhance a resolution of output frequency. In addition, a phase truncation is also not required.

To sum up, it is fully expected that the combination of DSM signal and DDS allows a fine frequency resolution even with simple circuit. In fact, simulation results with second order modulated signal demonstrated syntheses at extremely low frequencies. Moreover, analyses of these simulated outputs reveals that the high-frequency switching waveform of output has an almost purely sinusoidal component when generating frequency is low with respect to the clock frequency. Therefore, proposed technique is meaningful for applications which require synthesizing low frequencies and varying a output frequency continuously.

\section{REFERENCES}

[1] D.A. Johns and D.M. Lewis, "Design and analysis of Delta-Sigma based IIR filters," IEEE Trans. Circuits and Systems - II, vol. 40, no. 4, pp. 233 240, 1993.

[2] Fujisaka H.,Sakamoato M. and Morisue M., "Bit-stream signal processing circuits and their application," IEICE Trans. Fundamentals, vol. E85-A, no. 4, pp. 853-860, 2002.

[3] Murahashi Y., Doki S. and Okuma S.,"Hardware realization of novel pulsed neural networks based on delta-sigma modulation with GHA learning rule," in Proc. IEEE APCCAS'02, Singapore, Oct. 2002, vol. 2, pp. $157-162$.

[4] M. J. Flanagan and G. A. Zimmerman, "Spur-reduced digital sinusoid synthesis," IEEE Trans. Communications, vol. 43, pp. 2254-2262, July 1995.

[5] P. O'Leary and F. Maloberti, "A Direct-Digital Synthesizer with Improved Spectral Performance," IEEE Trans. Communications, vol. 39, no. 7, pp. 1046-1048, July 1991. 